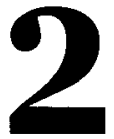

\title{
EL MODELO DE EXCELENCIA EN LA EFQM Y SU APLICACIÓN PARA LA MEJORA DE LA CALIDAD DE LOS CENTROS EDUCATIVOS
}

\author{
(THE EXCELLENCE MODEL OF EUROPEAN FOUNDATION FOR QUALITY \\ MANAGEMENT AND ITS APPLICATION FOR IMPROVING SCHOOL QUALITY)
}

Catalina Martínez Mediano

Nuria Riopérez Losada

Universidad Nacional de Educación a Distancia

\section{RESUMEN}

El presente trabajo expone las características del Modelo de Excelencia de la Asociación Europea para la Gestión de Calidad (EFQM), para su aplicación a los centros educativos. Revisa el concepto de calidad en la educación y los principios de la Calidad total, fundamentales para el desarrollo de los modelos de excelencia. Desde su creación en 1991, la Comisión Europea, la Organización Europea para la Calidad y la Asociación Europea para la Gestión de la Calidad, han promovido su utilización en las organizaciones empresariales y de otro tipo, incluidas las educativas, para la introducción de los principios de Gestión de Calidad Total. Las administraciones públicas promueven la aplicación del Modelo. Asimismo, organismos privados como la Confederación Española de Centros de Enseñanza, el Club Gestión de Calidad (CGC) y la EFQM, promueven su aplicación en los centros educativos, apoyados desde la administración pública que supervisan su aplicación y entrega premios y reconocimientos.

\section{ABSTRACT}

The present work exposes the characteristics of the European Foundation for Qualitiy Management (EFQM) Excellence Model, and its application to educational centers. It revises the concept of quality in education and the Total Quality Principles. From their creation in 1991, the European Commission, the European Organization for Quality and the European Association for Quality Management, have promoted their use in managerial organizations, including the educational ones, to introduce the principles of TotalQuality Management. Public Administrations and private organisms, as the Spanish Confederation of Centers of Teaching, and the Club of Quality Management (CGC) and the EFQM, promote the application of the 
Model in the educational centers. Public Administrations supervise their application and give prizes and recognitions.

\section{EL CONCEPTO DE CALIDAD DE LA EDUCACIÓN}

El concepto de "calidad de la educación", como meta de toda institución educativa, asume un enfoque global de gestión de la institución hacia la consecución de metas de calidad para todos y con la colaboración de todos los implicados en la institución educativa. La calidad conseguida tiene que ver con la calidad de las metas pretendidas, concretadas en objetivos, criterios y estándares que guíen su realización y la comprobación de su consecución.

La calidad en la educación se ha definido mediante un sistema de coherencias múltiples cuyas notas son funcionalidad, eficacia y eficiencia. La funcionalidad se entiende como la respuesta dada por el sistema educativo a las necesidades de formación de la sociedad. Estas necesidades son recogidas en los Sistemas Educativos y deben ser atendidas en los centros educativos, a través de los proyectos educativos para el desarrollo del plan de estudios y la formación de los alumnos. Una organización será eficaz si logra las metas educativas de calidad que se había propuesto, y será eficiente si hace un uso correcto de sus recursos, tanto personales como económicos y materiales, para conseguirlas.

La calidad abarca a todas las funciones y actividades de la institución y debe estar vinculada a las necesidades relevantes de la sociedad en un ámbito y contexto dado. Las necesidades y los objetivos, que orientarán la elaboración de los programas, la realización de los procesos, de los productos y de los servicios deben estar especificados, para dar respuesta a dichas necesidades, si bien a veces existen necesidades que se hacen explícitas a posteriori, con lo que su satisfacción aportará valor añadido sobre lo especificado. Desarrollar la calidad dentro de la organización, supone contar con un Sistema de Gestión de Calidad y estar formado en los modelos de evaluación de programas y centros, y sus procedimientos, así como en los modelos de excelencia.

\section{EL MODELO DE EXCELENCIA DE LA EFQM}

El Modelo de Excelencia de la EFQM fue presentado en 1991 y revisado en 1999 y en el 2003. Desde su creación, ha mostrado su eficacia como sistema de gestión de las organizaciones, a la vez que como instrumento para la autoevaluación y, además, se utiliza como marco para la valoración de los Premios Europeos a la Calidad, y, asimismo, para los Premios Nacionales a la Calidad. El Modelo está alineado con los principios de la calidad total. El modelo EFQM de excelencia es propiedad intelectual de la European Foundation for Quality Management, y se puede encontrar información sobre el mismo en internet: www.efqm.org. En la actualidad es el modelo más utilizado de evaluación de la excelencia por las organizaciones em- 
presariales europeas. El presente artículo se basa, entre otros documentos, en los que pueden encontrarse en la página web de la EFQM.

Independientemente del sector, tamaño, estructura o madurez, las organizaciones necesitan establecer sistemas apropiados de gestión. El Modelo de Excelencia de la EFQM es una herramienta práctica para ayudar a las organizaciones mediante la medición, en su camino hacia la excelencia, ayudándole a comprender sus lagunas y estimulando la búsqueda de soluciones. Los conceptos de excelencia están alineados con los principios de calidad total.

Las organizaciones utilizan el Modelo de Excelencia como fundamento para la operativización de las metas a conseguir, desde la planificación de sus procesos, su realización y la autoevaluación para la revisión de su proyecto. Por lo tanto, el modelo europeo de excelencia puede ser utilizado como:

- Herramienta para la autoevaluación.

- Modo de comparar las mejores prácticas entre organizaciones.

- Guía para identificar las áreas de mejora.

- Base para un vocabulario y estilo de pensamiento común.

- Estructura para los sistemas de gestión de las organizaciones.

El Modelo de Excelencia de la EFQM es un marco no prescriptivo basado en nueve criterios, cinco de ellos "Facilitadores o agentes" y cuatro "Resultados". Los criterios "Agentes" se refieren a lo que una organización hace. Los criterios "Resultados" se relacionan con lo que la organización logra. Los Resultados son causados por los "Agentes" y se mejoran mediante la retroalimentación.

El modelo, que reconoce que hay muchos enfoques para lograr la excelencia sostenible en todos los aspectos de actuación, se basa en la premisa de que:

"Los resultados excelentes con respecto a la realización de sus metas, la satisfacción de sus clientes, su personal y la sociedad se logran a través del liderazgo, conduciendo la política y la estrategia, a través de su personal, sus socios y recursos y sus procesos". Modelo.

Podemos ver, en el siguiente diagrama, las relaciones de los criterios en el

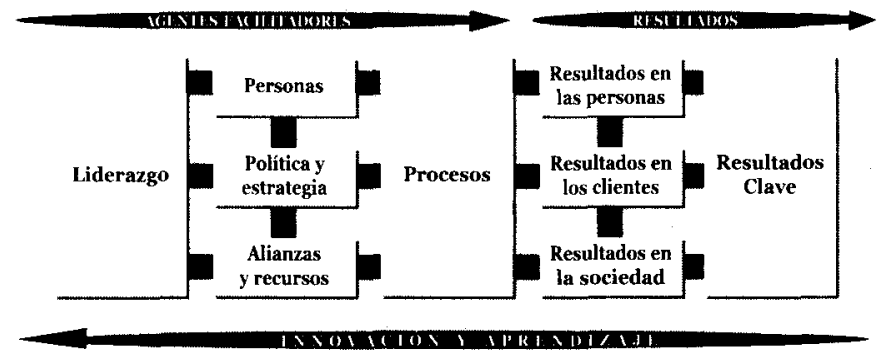

Figura 1. Diagrama del Modelo de Excelencia de la EFQM' 
Las flechas enfatizan la naturaleza dinámica del modelo. Muestran la innovación y el aprendizaje organizacional para mejorar los agentes que a su vez llevan a la mejora de los resultados.

\subsection{El Modelo de Excelencia europeo aplicado a los centros educativos}

Las administraciones educativas, los sistemas educativos y las instituciones educativas públicas y privadas participan del interés por el movimiento de la calidad total, para el logro de la excelencia, a través de la implicación de todos los miembros de la institución, en sus distintos niveles de responsabilidad, en los procesos de gestión y autoevaluación para la mejora y rendimiento de cuentas a la sociedad.

La Comisión Europea, desde 1997, promueve la utilización del Modelo EFQM para la evaluación de instituciones educativas, y son muchas las administraciones educativas públicas que lo han adaptado. En España, desde esa fecha, el Club Gestión de Calidad (CGC), representante en España de EFQM, con el Instituto de Técnicas de Estudio de la Confederación Española de Centros de Enseñanza (ITE, CECE) y el Ministerio de Educación, Cultura y Deporte (MECD), hicieron una adaptación del modelo para su aplicación en los Centros Educativos, actualizada en el 2001 que ha sido revisada en años posteriores.

La finalidad del modelo aplicado al ámbito educativo es conocer la realidad del centro mediante la autoevaluación, y reflexionar sobre ella, lo que posibilitará elaborar planes y estrategias de mejora. Estas dos características, junto con la implicación de todos en el proceso de autoevaluación y la toma de decisiones, se consideran factores que inciden en la mejora de la calidad de los servicios que el Centro Educativo presta a la sociedad. El modelo define nueve factores críticos para el buen funcionamiento de la organización a los que denomina criterios, fundamentados en elementos o indicadores. La comprobación sistemática de la existencia o no de dichos indicadores en la institución, como procedimiento para la autoevaluación, permite tener un conocimiento del funcionamiento del centro basado en hechos, con el fin de elaborar proyectos y planes de mejora en coherencia con las necesidades detectadas. La utilización de la autoevaluación para la recogida de información, como herramienta de mejora continua, permitirá avanzar en el logro de la política y estrategia del centro, concretados, entre otras cosas, en los objetivos de aprendizaje y formación de los alumnos, teniendo en cuenta la formación del personal y los recursos necesarios hacia la excelencia.

\subsection{Los conceptos fundamentales de excelencia}

Para aumentar al máximo los beneficios de adoptar el Modelo de Excelencia de la EFQM, el equipo de dirección debe asegurarse de que se encuentra cómodo con estos conceptos y son totalmente entendidos y aceptados; de lo contrario será difícil, y carente de sentido, alcanzar el progreso mediante la adopción del modelo. 
Los conceptos de excelencia adaptados a la Educación, quedan del modo siguiente:

- Orientación al cliente. La excelencia se consigue creando valor sostenible al cliente. El cliente, es la persona que se beneficia directamente de las actividades de la organización, que en el caso de los centros educativos es el alumno, su familia y la sociedad. El centro ha de identificar a sus clientes, sus necesidades y expectativas para satisfacerlas. La excelencia depende del equilibrio y satisfacción de las necesidades de todos los clientes relevantes, incluidas las personas que trabajan en la organización, los que solicitan sus servicios, los proveedores y la sociedad en general, así como todos aquellos con intereses, de un tipo u otro, en la organización.

- Liderazgo y constancia de propósito. El liderazgo ayuda a conseguir la excelencia si la visión y el propósito están alineados en la organización. Los líderes de una organización comprometida con la excelencia deben contribuir a la definición de la visión, la misión, la estrategia y los valores para conseguirla, para satisfacer las necesidades del cliente, reorientándola a la luz de los resultados y las nuevas necesidades, y favoreciendo la creación de un entorno en el cual la organización y las personas que la integran puedan alcanzar la excelencia.

- Orientación hacia los resultados. La excelencia se logra cuando los resultados satisfacen a todos los grupos implicados en la organización. Las mejoras propuestas deben estar fundamentadas en la información fiable, que incluye los hechos y las percepciones de los miembros de la institución y de los clientes.

- Desarrollo, implicación y reconocimiento de las personas. La excelencia se maximiza mediante la contribución al desarrollo, e implicación de las personas que trabajan en una organización en los procesos de mejora, mediante el trabajo en equipo. El potencial de cada una de las personas que trabaja en una organización debe ser desarrollado, y ello se favorece cuando se comparten los valores, y existe confianza mutua, lo cual anima a la involucración de todos hacia el logro de los objetivos de la organización.

- Gestión por procesos y hechos. La excelencia se consigue a través de la gestión con un enfoque de procesos y hechos de una forma sistémica. Todas las actividades deben estar interrelacionadas y ser gestionadas como un sistema, con la comprensión e implicación de todos los miembros de la institución. La institución consigue mejores rendimientos cuando trabaja en torno a procesos tendentes a dar respuesta a las necesidades detectadas. El término "proceso" se define como: "Conjunto de actividades mutuamente relacionadas o que interactúan, las cuales transforman elementos de entrada en resultados". Los procesos suelen ser interdepartamentales, por lo que 
se debe tener en cuenta las áreas, departamentos y personas implicadas para planificarlos y llevarlos a la práctica, y debe ser posible comprobar su realización mediante la utilización de un sistema de medición para reunir información y datos con el fin de analizar el desempeño del proceso y su trayectoria hacia la excelencia, buscando la mejora continua.

- Desarrollo de alianzas. La excelencia se consigue desarrollando y manteniendo alianzas que añadan valor en las personas, instituciones y organizaciones que suministran productos, servicios o conocimientos al Centro Educativo. Los proveedores, en una organización que persigue la excelencia, deben convertirse en socios, basándose en unas relaciones de confianza, que contribuyan a añadir valor. La organización trabaja de un modo más efectivo cuando comparte con sus socios el conocimiento en apoyo de su política y estrategia y del eficaz funcionamiento de sus procesos y de su personal.

- Proceso continuo de aprendizaje, innovación y mejora. La excelencia se consigue desafiando el "statu quo" y haciendo realidad el cambio aprovechando el aprendizaje para crear innovación y oportunidades de mejora. Las decisiones a tomar y los proyectos consecuentes deben derivarse del conocimiento del funcionamiento de la organización. Y este se basa en la revisión de los resultados, en coherencia con los criterios previos utilizados en la definición de los proyectos y programas, como son la misión, la política y la estrategia, y los elementos que la determinan: la formación del personal, las colaboraciones y utilización de los recursos, la determinación de los procesos, la planificación y realización de los mismos y su evaluación, subrayando la importancia de la retroalimentación, basada en la recogida de información fidedigna que permita introducir cambios, innovaciones, y oportunidades de mejora.

- Responsabilidad social de la organización. La excelencia se consigue excediendo el marco legal mínimo en el que opera la organización y esforzándose por entender y dar respuesta a las expectativas de los grupos de interés y de la sociedad. Los miembros de cualquier organización han de comportarse de acuerdo con una ética que otorgue significado a las acciones individuales más allá del que pueda derivarse de las normas o requisitos legales para dar respuesta a las necesidades y expectativas de los grupos de interés y de la sociedad, especialmente en el ámbito de lo educativo, cuya repercusión en las personas y en la sociedad es fundamental.

\subsection{Los criterios del Modelo de Excelencia Europeo}

El Modelo está integrado por nueve criterios frente a los cuales se evalúa el progreso de la organización hacia la excelencia, -cinco criterios facilitadores y cuatro resultados-. Cada criterio define y explica su significado, y se fundamenta por un número de elementos que, en forma de pregunta para contrastar con la práctica de la organización, ayuda a la autoevaluación del centro. 
Los criterios agentes definen lo que pretende conseguir la organización mediante el liderazgo, la política y estrategia, el personal, los colaboradores y recursos, y los procesos:

1. Liderazgo: Los líderes excelentes desarrollan y facilitan la consecución de la misión y la visión, desarrollan los valores y sistemas necesarios para que la organización logre un éxito sostenido y hacen realidad todo ello mediante sus acciones y comportamientos, reorientan la organización cuando es necesario. (Integrado por cinco elementos: sobre el desarrollo de la misión, visión, valores y principios éticos, implicación del personal para garantizar el desarrollo, implantación y mejora continua. Interactúan con clientes y colaboradores, refuerzan la cultura de excelencia en las personas, definen e impulsan el cambio en la organización).

2. Política y estrategia: Las organizaciones excelentes implantan la visión, la misión y los valores y desarrollan la estrategia centrada en los grupos de interés, y desarrollan y despliegan las políticas, planes, objetivos y procesos para hacer realidad la estrategia de la organización. (Integrado por cuatro elementos: La política y estrategia se basa en las necesidades y expectativas actuales y futuras, en la información de indicadores de rendimiento, investigación, aprendizaje, actividades externas, se desarrolla, revisa y actualiza, se comunica y despliega mediante un esquema de procesos clave).

3. Personas: Las organizaciones excelentes gestionan, desarrollan y hacen que aflore todo el potencial de las personas que las integran. Fomentan la justicia y la igualdad e implican a las personas. (Integrado por cinco elementos: Planificación, gestión y mejora de los recursos humanos. Identificación, desarrollo y mantenimiento del conocimiento y la capacidad de las personas. Implicación y asunción de responsabilidades por las personas. Existencia de diálogo. Reconocimiento y atención a las personas).

4. Alianzas y recursos: Las organizaciones excelentes planifican y gestionan las alianzas externas, proveedores y recursos internos en apoyo de su política y estrategia y del eficaz funcionamiento de sus procesos. Establecen un equilibrio entre las necesidades actuales y futuras de la organización, comunidad y medio ambiente. (Integrado por cinco elementos: Gestión de alianzas externas. Gestión de los recursos económicos y financieros. Gestión de edificios, equipos y materiales. Gestión de la tecnología. Gestión de la información y del conocimiento).

5. Procesos: Las organizaciones excelentes diseñan, gestionan y mejoran sus procesos para satisfacer plenamente a sus clientes y grupos interesados, y generar cada vez mayor valor para ellos. (Integrado por cinco ele- 
mentos: Diseño y gestión sistemática de los procesos. (Investiga las necesidades de los clientes y del personal. Elabora el plan de educación y formación del centro. Plan de enseñanza, de evaluación de los alumnos y de los procesos de enseñanza-aprendizaje. Desarrollo de actividades extraescolares y complementarias. Procesos de dirección en todos los niveles. Relaciones y comunicación con los clientes e interesados. Gestión de nuevos alumnos) (Comprobación de mejora continuada de los resultados, en coherencia con los requerimientos y necesidades explícitas e implícitas. Establece indicadores sobre los resultados de los procesos y fija objetivos de mejora. Gestión y apoyo a la implantación de cambios a través del control del proyecto, verificación, formación y revisión. Revisa los procesos clave para asegurar su progreso y garantizar su éxito).

\section{Los criterios de los resultados}

Los criterios del bloque de los resultados tienen como finalidad conocer lo que ha conseguido el Centro Educativo. A priori señala los resultados que desea obtener el centro. Son los siguientes:

6. Resultados en los clientes: Las organizaciones excelentes miden de manera exhaustiva los resultados que esperan se alcancen en el alumno, $y$ su familia, en relación con lo que está definido en su misión. (Percepción de padres y alumnos de la educación y formación que da el centro). (Integrado por dos elementos: Medidas de percepción e Indicadores de rendimiento).

7. Resultados en el personal: Las organizaciones excelentes miden de manera exhaustiva los resultados que esperan que se alcancen en las personas que la integran. (Expectativas generadas por el Proyecto Educativo del centro (enfoque metodológico, exigencia). Satisfacción por la metodología de enseñanza y formativa. (Resultados académicos). (Integrado por dos elementos: Medidas de percepción e Indicadores de rendimiento)).

8. Resultados en la sociedad: Las organizaciones excelentes miden de manera exhaustiva los resultados que esperan que se alcancen en la sociedad. (Integrado por dos elementos: Medidas de percepción e Indicadores de rendimiento).

9. Resultados clave de la organización: Las organizaciones excelentes miden de manera exhaustiva los resultados que esperan que se alcancen con respecto a los elementos clave de su política y estrategia. (Indicadores de los resultados educativos y de otros servicios prestados por el centro). (Integrado por dos elementos: Medidas de percepción e Indicadores de rendimiento).

A cada uno de los nueve criterios se le asigna una puntuación para compro- 
bar la situación de la organización mediante la autoevaluación, y también para evaluar las solicitudes al Premio y reconocimiento de Calidad. Los valores que han sido adoptados por el MECD para su aplicación a los Centros Educativos son los siguientes: Criterio 1=12; Criterio $2=10$; Criterio $3=7$; Criterio 4=7; Criterio 5=14; Criterio $6=15$; Criterio $7=11$; Criterio $8=10$; Criterio $9=14$.

\subsection{Los pasos para la implantación del Modelo}

El camino hacia la excelencia se basa en el compromiso por la mejora continua, la autoevaluación, la gestión de las buenas prácticas y la disciplina de planificación. La organización, en ese compromiso hacia la mejora continua, debe preguntarse si cuenta con un plan para la mejora, y si no es así, debe iniciar los pasos para la autoevaluación y, a partir de ello, la elaboración de planes de mejora.

\subsubsection{Etapas para la implantación del Modelo}

Para la implantación del modelo, teniendo en cuenta los conceptos de la excelencia, se deben dar los siguientes pasos:

\section{- Etapa previa}

1. Liderazgo y compromiso del equipo directivo del centro y de las autoridades educativas con la mejora.

2. Sensibilización e información del personal del centro, profesores y personal de administración y servicios.

3. Facilitación de la implantación mediante la ayuda de Expertos Externos en el Modelo.

4. Constitución de un Equipo de Calidad integrado por la dirección y personas del Centro interesadas.

\section{- Etapa de autoevaluación del centro}

5. Realización de la autoevaluación por el Equipo de Calidad. Aplicación de los instrumentos de recogida de información del Modelo, mediante el Cuestionario y/o el Formulario. Análisis de los datos y elaboración del informe de resultados. Indicación de los Puntos Fuertes y de las Áreas de Mejora.

6. Priorización de las Áreas de acuerdo con criterios de incidencia en los resultados clave de la organización, alineados con la política y la estrategia del centro.

7. Presentación de los resultados, por parte del Equipo Directivo, al Claustro y al Consejo Escolar, para concretar el Plan de Mejora para el Centro.

\section{- Etapa de elaboración del plan de mejora}

8. Constitución de los equipos de mejora en función de los temas, nombramiento de los responsables de los proyectos y colaboradores.

9. Elaboración de los Planes de Mejora, en coherencia con las necesidades detectadas, y alineados con los Proyectos Institucionales del Centro Educa- 
tivo, señalando sus responsables, ámbitos de aplicación, temporalización y criterios para su seguimiento y evaluación.

10. Presentación de los Planes de Mejora para su conocimiento y aprobación al Claustro y al Consejo Escolar.

11. Determinación y planificación de los procesos y recursos necesarios para realizarlos.

\section{- Etapa de aplicación del plan de mejora}

12. Ejecución y seguimiento de los procesos de mejora, medición de resultados.

13. Difusión y discusión de los resultados.

14. Verificación de los resultados de los Planes de Mejora, y realización de una nueva autoevaluación.

15. Adopción del principio de innovación, aprendizaje y mejora continua en el Centro.

\subsubsection{La autoevaluación}

La autoevaluación es la herramienta fundamental del Modelo de Excelencia, entendida como un examen global y sistemático de las actividades y resultados de una organización que se compara con un Modelo. Mediante el proceso de la autoevaluación se pretende conseguir una comprensión detallada del Centro Educativo, buscando realizar un diagnóstico sobre cual es la situación actual del centro, señalando los puntos fuertes y las áreas de mejora. A partir de este diagnóstico se elaboran propuestas de mejora, objetivas, concretas y conseguibles en un plazo determinado, arbitrando un seguimiento de dicho plan de mejora, para ayudar a su implantación y valoración de logro mediante la autoevaluación, introduciéndose, de este modo, en el centro, una dinámica de mejora continúa mediante la planificación, aplicación y valoración de proyectos de mejora innovativos en una tendencia de mejora sostenible. Aunque la autoevaluación suele ser aplicada al conjunto de la organización, también puede evaluarse un proyecto concreto, un departamento o cualquier otra unidad de servicio.

La autoevaluación como camino hacia la mejora puede verse con la metáfora del viaje, en las siguientes etapas:

1. Evaluar para saber dónde nos encontramos. Para determinar la situación actual, podemos hacerlo mediante la autoevaluación de la organización, o Centro Educativo. El proceso en sí de la autoevaluación puede ayudar a la organización a producir un marco informativo del centro.

2. Definir las prioridades del centro. Para alinear la organización con la estrategia, necesita entender sus fuerzas actuales y las áreas de mejora. Los conceptos fundamentales de excelencia son la expresión más tangible o concreta, de la excelencia, y puede compararlos con los que utiliza su propia organización. 
3. Identificar las necesidades de mejora. La herramienta de la autoevaluación del Modelo de Excelencia de la EFQM puede ayudar a proporcionar un mapa para las personas de la organización, con la finalidad de conocer dónde se necesita mejorar.

4. Identificar cómo mejorar. Aprender de las organizaciones que tiene alrededor a través del "benchmarking", las mejores prácticas, y de la investigación. Identificar las buenas prácticas de los otros. Puede tener procesos de referencia, de organización y/o de medida... pero primero debe desarrollar una estrategia de referencia que ayude a dirigir sus esfuerzos.

El Modelo establece el esquema de determinar los Resultados a lograr, planificar y desarrollar los Enfoques -Approach-, Desplegar -Deployment- los enfoques, Evaluar -Assessment- y Revisar los enfoques y el despliegue (REDER, o RADAR, actualización del ciclo PDCA: Plan Do Check Act), como método para la autoevaluación, para ver los valores dados por los evaluadores a cada elemento de los criterios Agentes y Resultados.

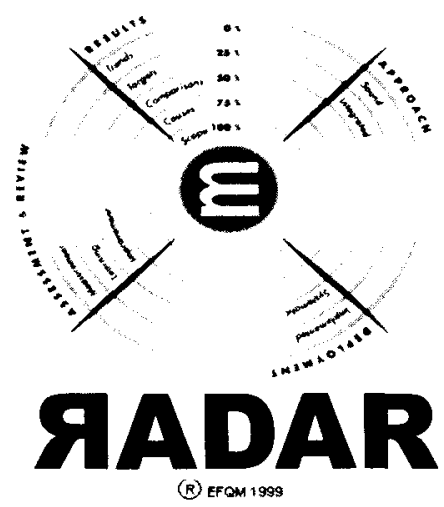

Figura 2. La matriz RADAR para la autoevaluación

Criterios agentes: Hacen referencia a lo que hace el centro para conseguir las metas pretendidas. Todos los elementos tienen el mismo peso en este bloque. La valoración dada a cada pregunta debe tener en cuenta:

- El enfoque: Debe abarcar lo que una organización ha planificado hacer y las razones para ello. Es el diseño del plan para lograr los resultados, que debe estar:

Fundamentado: el enfoque estará sólidamente fundamentado en las teorías y estrategias. Tendrá una lógica clara, con procesos bien definidos y desarrollados y una clara orientación hacia las necesidades de todos los grupos de interés.

Integrado: el enfoque estará integrado, es decir, apoyará la política y la estrategia y, cuando así convenga, estará vinculado a otros enfoques. Anticipatorio: previendo los posibles problemas y dificultades. 
- El despliegue: se ocupa de lo que hace una organización para desplegar el enfoque. Se implantará en las áreas relevantes, de un modo sistemático. Debe contener los objetivos, contenidos, estrategias del enfoque e informar de ello a quienes lo deben conocer: equipo de profesores, alumnos, padres. Y desarrollarlo, aplicarlo, de acuerdo con el plan.

- La evaluación y revisión del enfoque y despliegue de manera sistemática, regular, integrada, para la mejora. Se centra en lo que hace una organización para evaluar y revisar el enfoque y el despliegue de dicho enfoque. El enfoque y su despliegue estarán sujetos a mediciones regulares, sus resultados se utilizarán para identificar, establecer prioridades para planificar e introducir cambios en implantar la cultura de la mejora continua en la organización.

Criterios resultados: hacen referencia a lo que ha conseguido o está consiguiendo el Centro. La valoración debe basarse en hechos reales. Y centrarse en la magnitud (nivel de excelencia de los resultados) y alcance (ámbito de aplicación). La valoración dada a cada pregunta debe tener en cuenta:

- La magnitud: grado de excelencia del resultado obtenido.

- Las tendencias: ¿qué ha obtenido en los últimos años? Una organización excelente mostrará tendencias positivas y/o un buen rendimiento sostenido.

- Las metas: los objetivos serán adecuados y se alcanzarán. ¿Qué quería conseguir y qué ha obtenido?.

- El rendimiento obtenido, que en una organización excelente será consecuencia de los enfoques, y bueno, comparado con el de otras organizaciones. Servirá para comparar los resultados del programa con otros programas similares.

- El alcance: referido al ámbito de aplicación, y a la importancia de los resultados obtenidos, áreas relevantes a las que atiende, que tiene que ver con la cobertura: ¿Cubren los resultados todas las áreas que se pretenderán alcanzar? Y la relevancia: ¿Son relevantes los resultados para los usuarios, incluidos el personal del centro?.

\subsubsection{Los instrumentos para la autoevaluación}

\subsubsection{El cuestionario}

El cuestionario para la autoevaluación del Centro Educativo, de acuerdo con el Modelo de Excelencia de la EFQM, es un instrumento sencillo, aconsejado para un primer acercamiento a la situación del Centro Educativo, a contestar de modo 
individual por un grupo representativo de todas las sensibilidades del centro. Deben ser contestados por el mayor número de personas relacionadas con la institución, pidiéndoseles que traten de ser objetivos y rigurosos en su respuesta. Es un instrumento útil para reconocer los éxitos del pasado así como para identificar el potencial de progreso del Centro hacia el futuro ${ }^{2}$. Está integrado por 108 preguntas, que deben responderse puntuando con los 4 valores.

\begin{tabular}{|c|l|l|}
\hline Puntuación & Progreso & \\
\hline 1 & $\begin{array}{l}\text { Ningún } \\
\text { avance }\end{array}$ & $\begin{array}{l}\text { - ninguna acción se ha realizado aún. } \\
\text { - quizás algunas ideas buenas que no se han concretado. }\end{array}$ \\
\hline 2 & $\begin{array}{l}\text { Cierto } \\
\text { avance }\end{array}$ & $\begin{array}{l}\text { - parece que se está produciendo algo. } \\
\text { - análisis ocasionales que dieron lugar a ciertas mejoras. } \\
\text { - con algunas prácticas se han logrado resultados aislados. }\end{array}$ \\
\hline 3 & $\begin{array}{l}\text { Avance } \\
\text { significativo }\end{array}$ & $\begin{array}{l}\text { - clara evidencia de que se han planteado y tratado este tema de manera adecuada. } \\
\text { - existenes sistemáticas y frecuentes que logran mejoras. } \\
\text { aprovechado todo el potencial. }\end{array}$ \\
\hline 4 & $\begin{array}{l}\text { Objetivo } \\
\text { logrado }\end{array}$ & $\begin{array}{l}\text { - planteamiento muy excelente o resultado aplicado en su extensión máxima. } \\
\text { - solución o resultado que puede servir como modelo y resulta dificil pensar que } \\
\text { pueda ser mejorado. }\end{array}$ \\
\hline
\end{tabular}

Una vez que los cuestionarios han sido analizados se establece un perfil del Centro, en el que se reflejan las puntuaciones medias obtenidas en cada uno de los criterios del Modelo.

\subsubsection{El formulario}

Es otro de los instrumentos que ofrece el Modelo para la autoevaluación, que aporta sobre el cuestionario, una mayor exhaustividad, evidencias que fundamente las respuestas, y unos criterios más amplios para emitir las respuestas, facilitando aportar sugerencias, por cada uno de los criterios, a tener en cuenta para la elaboración de los planes de mejora de una manera más ajustada a las necesidades del centro.

Ambos instrumentos, contestados de modo individual, requieren de una reunión por parte del equipo de evaluación con los evaluadores, para llegar a consensos en las cuestiones que muestran divergencias en las valoraciones. Así, si las divergencias son inferiores al $25 \%$, se recomienda obtener valores medios, pero si las divergencias son superiores a este $25 \%$ hay que revisarlas y aportar argumentos explicando las puntuaciones otorgadas. La relación de puntos fuertes y áreas de mejora vienen determinadas por las valoraciones de la mayoría.

\subsubsection{La herramienta perfil V.4.0}

La herramienta Perfil, en su versión V.4.0, del Modelo EFQM de Excelencia, del 2003, ha sido desarrollada por el Club Gestión de Calidad, en una aplicación para su uso informatizado, y adaptada al ámbito educativo, con la colaboración de 
entidades como CECE, FERE-COVAL, Fundación Navarra para la Calidad e Institución Educativa SEK, que posibilita la autoevaluación de la organización con la participación de todos los miembros que deseen colaborar. Presenta una amplia explicación del Modelo EFQM de Excelencia, y de cada uno de los criterios que lo integran.

Para la autoevaluación se utiliza el instrumento del cuestionario, en un formato intermedio entre el cuestionario y el formulario, en el que el cuestionario muestra los ítems para ser valorados mediante una escala, y el formulario especifica que hay que aportar evidencias y explica con gran detalle cómo se deben emitir las valoraciones de los criterios agentes y resultados. En la aplicación de la herramienta Perfil, cada una de las preguntas aparecen en una pantalla de ordenador independiente, en la que se define el criterio, se muestra el diagrama del modelo y el criterio al que pertenece, además de "sugerencias", que ayuden al evaluador a comprender el sentido de la pregunta. La valoración de la pregunta se hace en torno a una escala de 0 a 100, debiendo aportar el evaluador evidencias que justifiquen la puntuación que otorga.

La herramienta permite visualizar los resultados de la autoevaluación mediante una serie de gráficos tales como diagramas de barras por puntos, o por porcentajes, Radar por puntos o por porcentajes, que permiten comparar las puntuaciones que está otorgando el evaluador con los valores de referencia del Modelo, informándole si ha completado, o no, el cuestionario. Las gráficas permiten comparar las puntuaciones obtenidas por la organización en el Modelo, con las puntuaciones máximas de referencia y las puntuaciones medias obtenidas por las organizaciones de su ámbito.
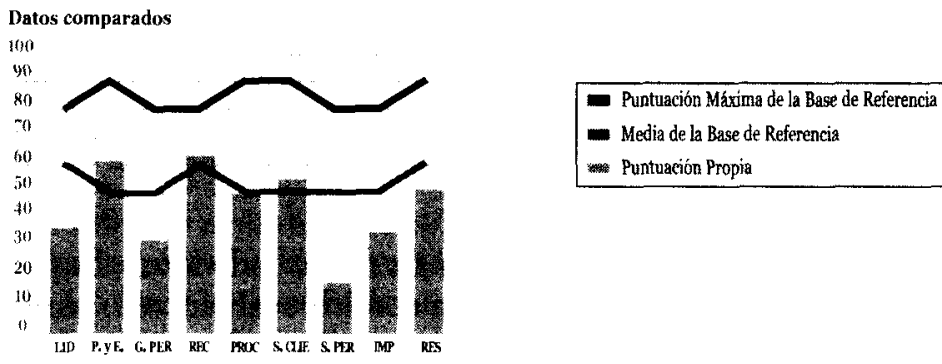

Figura 3. Diagrama de barras de la herramienta Perfil (Del Club Gestión de Calidad)

Perfil V.4.0, muestra, mediante el mapa de ejes, las relaciones existentes entre los diferentes subcriterios de distintos criterios. En la tabla siguiente mostramos los 11 ejes que recoge la herramienta perfil V.4.0, la relación de los ejes entre sí, y su correspondencia con los nueve criterios del Modelo de Excelencia de la EFQM. 


\section{MAPA DE EJES Y RELACIONES, Y CRITERIOS A LOS QUE PERTENECEN}

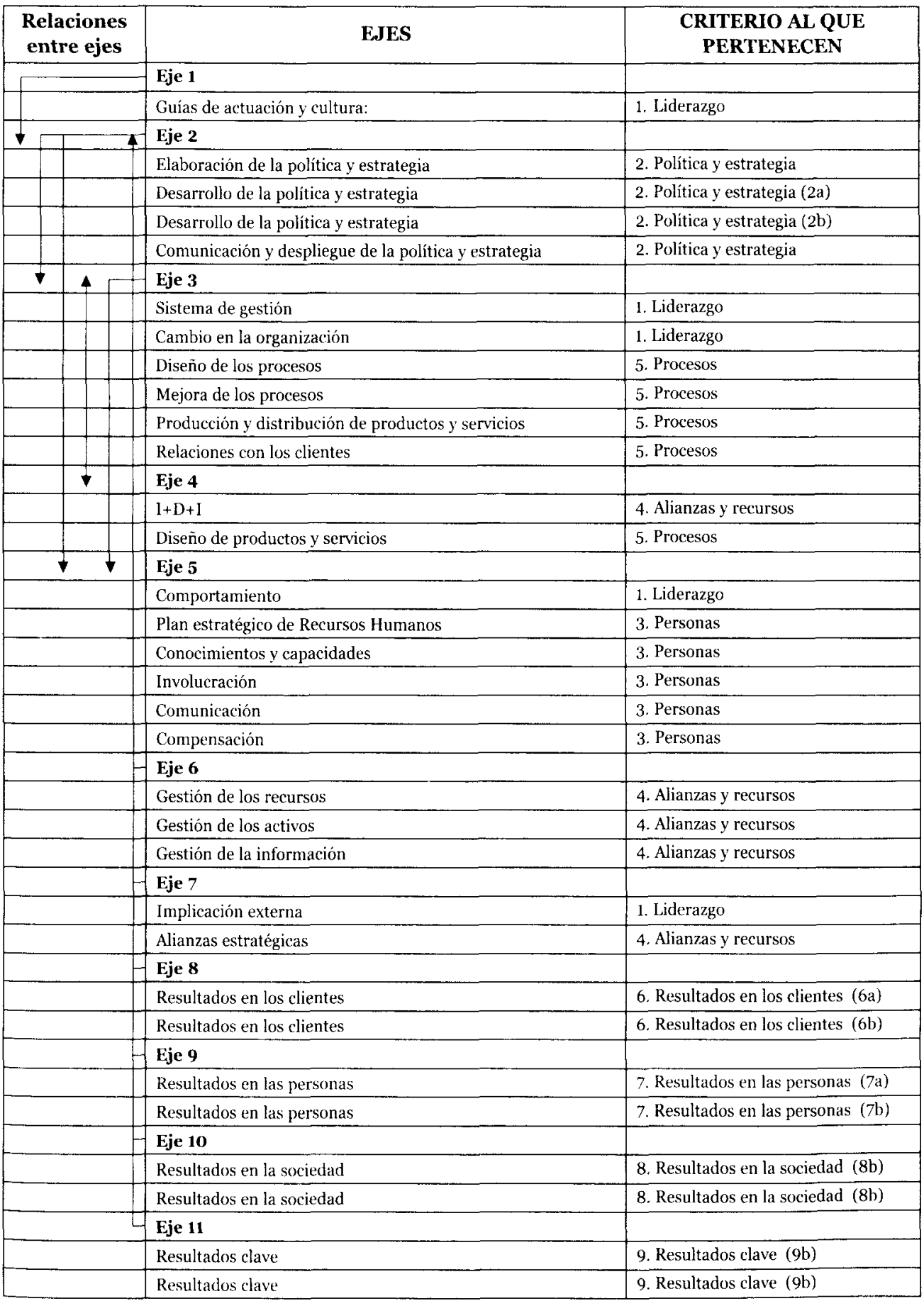




\section{LA ELABORACIÓN DE LOS PLANES DE MEJORA}

Continuando con el camino hacia la excelencia, ahora hay que aplicar las mejoras en coherencia con los resultados de la autoevaluación, mediante la elaboración de planes de mejora. Poner la excelencia en práctica significa priorizar, planear, realizar las mejoras y comprobar sus resultados.

1. Priorice las mejoras. No siempre se puede hacer todo a la vez. Es crucial revisar las áreas de mejora y determinar qué acciones se necesitan hacer primero. Por ejemplo, ¿qué mejoras tendrán mayor impacto en la organización? Se deben priorizar las acciones de mejora teniendo en cuenta la importancia, la urgencia y los recursos requeridos.

2. Incorpore las mejoras en sus planes de acción. Algunas mejoras importantes requerirán más planificación y recursos, para lo que será necesario organizar estas acciones a través de un enfoque más formal de gestión de proyectos.

3. Haga las mejoras. Una parte muy importante de los procesos de mejora es el cambio. Para mejorar debe cambiar. Para tener éxito en los proyectos de mejora es necesario involucrar, comprometer a todas las personas de la organización en perseguir la excelencia.

4. Compruebe los resultados. ¿Ha logrado sus objetivos? ¿Puede medir la mejora en su organización? En la organización se debe identificar la trayectoria del progreso mediante la autoevaluación en intervalos regulares frecuentes.

Un plan de mejora parte de los resultados de la autoevaluación que ha mostrado las fortalezas y las debilidades de la misión y la visión del centro, de las estrategias, de los valores, de los enfoques, de los despliegues y de los resultados. La autoevaluación, de este modo, realiza una función diagnóstica indicando las necesidades de mejora.

Los criterios para priorizar las áreas de mejora son los siguientes:

- La gravedad de las carencias detectadas.

- La factibilidad con la que se puedan emprender las acciones.

- La rentabilidad prevista en sus resultados, el impacto.

- Las valoraciones de los grupos implicados.

Una vez priorizadas y consensuadas las necesidades, se elaboran en coherencia los Planes de mejora. Un plan de mejora es un instrumento que parte de la autoevaluación, que ha marcado la línea base en la que se encuentra el centro, y sobre la que se deben promover las mejoras. El plan especifica los objetivos a conseguir de modo realista, concretos y evaluables, siendo necesario concretar un plan de seguimiento para constatar los logros. El seguimiento sirve a la institución como instrumento para el aprendizaje organizativo. 
La implantación del Modelo europeo en los centros educativos, tiene, entre otras, las siguientes ventajas:

- Supone incorporar un plan de calidad, como base para el desarrollo de mejora continua de la institución educativa.

- Fomenta y facilita la reflexión de todos los miembros de la comunidad educativa: profesores, padres, alumnos y colaboradores.

- Permite descubrir las debilidades y fortalezas de la institución, basadas en hechos y datos, fundamento de la propuesta de planes para la mejora continua.

- Genera actitudes positivas para dar respuesta a una sociedad con continuas necesidades y demandas de cambio e innovación.

\section{LOS NIVELES EFQM DE EXCELENCIA. LOS PREMIOS}

Los niveles de excelencia se crearon en 1992 a propuesta de la Comisión Europea, la Organización Europea para la Calidad (OEC) y la EFQM, para ofrecer a las organizaciones un reconocimiento común a toda Europa para cada una de las etapas que se contemplan en el camino hacia la excelencia. El proceso de solicitud proporciona a la organización una evaluación externa imparcial de la posición que ocupa frente al modelo EFQM, a la vez que le proporciona orientación en sus procedimientos de autoevaluación. Basándose en los informes de equipos de evaluadores acreditados, un Jurado selecciona a los galardonados, y entre éstos se entrega el premio europeo a la calidad al mejor de ellos, así como otros reconocimientos y menciones.

Los objetivos que se persiguen con los niveles EFQM de excelencia son:

- Ofrecer a las organizaciones oportunidades de reconocimiento, contemplando diferentes niveles de excelencia.

- Incrementar al máximo el número de organizaciones que aplican los principios del modelo EFQM para la mejora.

- Proporcionar informes independientes de las evaluaciones, para apoyar a las organizaciones en sus esfuerzos de mejora.

- Ofrecer productos y servicios prácticos que ayuden a las organizaciones a mejorar, desde un enfoque global, sus niveles de excelencia.

Se contemplan los siguientes niveles de excelencia:

1. Premio europeo a la calidad. Es el premio más prestigioso, (European Quality Award, EQA) y constituye el nivel superior. Se otorga anualmente desde 1992. Se dirige a organizaciones que han alcanzado estándares de calidad de prestigio internacional. Pueden optar al premio organizaciones miembros de EFQM y no miembros con independencia de su tamaño y sector, y existen diferentes categorías. El EQA se otorga sólo a organizacio- 
nes europeas. Este premio lo consiguen empresas que destacan mundialmente.

2. Reconocimiento a la excelencia. Se basa en una evaluación de la organización de acuerdo con el Modelo EFQM, de modo global, mostrando con este enfoque estructurado sus puntos fuertes y áreas de mejora, reconociendo el éxito de los esfuerzos por implantar los conceptos de excelencia y las buenas prácticas. Pueden optar miembros y no miembros de EFQM. Requiere que el candidato presente una memoria más breve que al premio europeo a la calidad, otorgando el Reconocimiento a aquellas organizaciones que alcancen una puntuación de 400 puntos o más, en el Modelo.

3. Compromiso con la excelencia. Con este nivel se pretende ayudar a las organizaciones que han iniciado el camino hacia la excelencia a comprender cuál es su nivel actual de rendimiento y establecer prioridades de mejora. Para obtener este reconocimiento, que se caracteriza por dos etapas, los candidatos deben realizar, primero, una autoevaluación global de su organización siguiendo los 9 criterios del Modelo, para tener una visión general de su funcionamiento en relación con el modelo. Como resultado, la organización obtiene una información sobre las áreas de mejora relevantes. En segundo lugar, la organización debe demostrar que ha implantado y desarrollado unas determinadas acciones de mejora.

Además de estos premios, para cuya obtención las organizaciones compiten entre sí, se han desarrollado reconocimientos basados en el Modelo EFQM que siguen el esquema "Sello", por el cual las organizaciones candidatas no compiten entre sí, sino que se consigue, para un período de tiempo renovable, al superar una puntuación determinada tras un proceso de evaluación siguiendo el Modelo EFQM. Los otorga la EFQM, y el Club Gestión de Calidad, representante de EFQM en España.

El procedimiento que se sigue es la Evaluación con el Modelo EFQM por parte de un Equipo de Evaluadores. Los organizadores del premio hacen pública la convocatoria anual, los candidatos preparan la memoria y presentan la solicitud para optar al premio o reconocimiento. El organizador del premio, mediante equipos de evaluadores externos independientes, acreditados, evalúa la candidatura, y un jurado convocado por la entidad organizadora de los premios, de entre los candidatos evaluados que cumplen con los criterios establecidos, y que han obtenido por los equipos de evaluadores la mayor puntuación, que se constituyen en finalistas, deciden quienes son merecedores de los premios.

La EFQM convoca los Premios Europeos a la Calidad, el Club Gestión de Calidad convoca los Sellos a la calidad, y las entidades certificadoras licenciatarias de Autoevaluación EFQM, reconocidas por el CGA, evalúan, y oficializan la autoevaluación EFQM de la organización para poder presentarse al premio "Sello", y las entidades certificadoras, evalúan, deciden y renuevan el premio. El Club Gestión de Calidad, propietaria de la marca Sello CGC, supervisa todo el proceso. 
Las categorías de los sellos son las siguientes:

- Sello Bronce (de 201 a 400 puntos en el Modelo EFQM, equivalente al Sello Calidad Europea).

- Sello Plata (de 401 a 500 puntos en el Modelo EFQM, equivalente al Sello Excelencia Europea, nivel de consolidación).

- Sello Oro (más de 500 puntos en el Modelo EFQM, equivalente al Sello Excelencia Europeo nivel de Excelencia).

El Ministerio de Educación y Cultura creó el Premio a la Calidad en Educación en 1998, con el fin de promover la mejora de la educación a través de la mejora de la gestión de los centros, extender los conceptos de excelencia en la gestión, y el Modelo EFQM como marco orientador a los centros, para facilitar su uso como referente común que permita el intercambio de experiencias y la difusión de las buenas prácticas de gestión educativa.

El premio tiene cuatro modalidades según que se dirija a: 1) los centros de titularidad pública de enseñanzas regladas de educación infantil y primaria, 2) centros de titularidad pública que impartan educación secundaria, formación profesional, enseñanzas artísticas, educación de adultos y escuelas oficiales de idiomas, 3) centros de educación especial y 4) centros docentes de titularidad privada que impartan las enseñanzas anteriormente mencionadas.

Los centros que se presenten al premio deben elaborar una memoria en la que en su primer apartado describan el centro: su historia, contexto y entorno, organigrama y su funcionamiento tomando como referente el Modelo Europeo de Excelencia adaptado a los centros educativos, o cualquier otra de las formas de evaluación propuestas en la convocatoria. Las memorias son evaluadas por un grupo de personas seleccionadas por el Ministerio. Los resultados de la evaluación son, a su vez, valorados por un jurado nombrado por el Ministro de Educación, al que le presenta una propuesta definitiva para la concesión de los Premios. El premio consiste en una cantidad económica, un diploma y una medalla, pudiendo concederse menciones honoríficas. Las organizaciones que obtienen este reconocimiento a la excelencia se consideran bien gestionadas, pudiendo utilizar este reconocimiento con fines comerciales y de promoción.

\section{LA APLICACIÓN DEL MODELO DE EXCELENCIA DE LA EFQM A LOS CENTROS EDUCATIVOS}

Mostramos una síntesis de varias experiencias de aplicación del Modelo de Excelencia de la EFQM a Centros Educativos, utilizando como herramienta para la autoevaluación mediante el cuestionario, el formulario y la aplicación informática Perfil V.4.0, con la finalidad de reflexionar sobre la situación del Centro e iniciar un proceso de mejora continua, y no, en esta primera fase, para la evaluación externa. Aportamos los resultados obtenidos en centros de educación infantil, primaria y secundaria y de formación profesional, tras la aplicación del cuestionario, y las pro- 
puestas para la elaboración de Planes de mejora que se derivan de los resultados. Esta información procede de un curso que dirige una de las autoras de este artículo, Catalina Martínez, del Programa de Formación del Profesorado de la UNED, protegiendo la confidencialidad de los centros.

\subsection{Experiencia en el Centro Educativo 1}

El objetivo pretendido en la aplicación del Modelo, en este Centro, es realizar un autodiagnóstico de su situación, para conocer sus puntos fuertes y áreas de mejora, elaborar planes de mejora en coherencia con las necesidades detectadas, implementarlos, verificar sus efectos, y recoger información de una manera continua, consolidando las mejoras conseguidas y la cultura de mejora continua en el centro.

Para ello se informó al claustro del centro, y se pidió su colaboración, consiguiendo que el cuestionario fuera contestado por 22 profesores del centro. Los resultados obtenidos muestran el siguiente perfil del Centro:

\begin{tabular}{|ll|l|}
\hline \multicolumn{2}{|c|}{ Perfil del Centro 1 } \\
\hline Criterios & Valoración Media \% \\
\hline $1 . \quad$ Liderazgo & 46 \\
\hline $2 . \quad$ Política y Estrategia & 35 \\
\hline $3 . \quad$ Personal del centro & 45 \\
\hline $4 . \quad$ Colaboradores y Recursos & 42 \\
\hline $5 . \quad$ Procesos & 43 \\
\hline $6 . \quad$ Resultados en Usuario & 52 \\
\hline $7 . \quad$ Resultados en Personas & 46 \\
\hline $8 . \quad$ Resultados en Sociedad & 37 \\
\hline $9 . \quad$ Resultados Clave Centro & 45 \\
\hline Total & 43,44 \\
\hline
\end{tabular}

Los resultados del cuestionario aportan información suficientemente detallada sobre cada uno de los 9 criterios, para orientar a la elaboración de los planes de mejora. Así, por cada criterio se puede recoger, además de la puntuación general, los subcriterios más necesitados de mejora y los mejor valorados. Entre los puntos fuertes y áreas de mejora recogemos la siguiente síntesis:

\section{Liderazgo}

El equipo directivo es accesible a todo el personal. Sin embargo, es necesaria una normativa para favorecer la asunción de responsabilidades, la valoración y la incentivación de la participación.

\section{Política $y$ estrategia}

Se ve la necesidad de definir la política y la estrategia, y alinear con ellas todos 
los procesos del centro, y concretarlos con objetivos claros, estrategias para llevarlos a cabo, y determinar procedimientos de seguimiento de logro e indicadores para su medición y valoración. Con responsables y coordinación entre todos. Asimismo, es conveniente mejorar la proyección de la imagen externa del centro, para atraer a más alumnos, y colaboraciones, y ampliar nuestras ofertas de estudios con modalidades a distancia.

\section{Personal}

Se ve necesario elaborar planes de formación del profesorado, especialmente en nuevas tecnologías y enfoques didácticos. También es necesario diseñar y aprobar un plan de evaluación interna del profesorado, junto con un plan para la incentivación del personal con iniciativas para conseguir convenios y proyectos beneficiosos para el centro.

\section{Colaboradores y recursos}

Es necesario obtener recursos mediante concursos a proyectos, premios y colaboraciones de las administraciones locales, y colaborar con los centros educativos del entorno.

\section{Procesos}

Funciona bien la designación de responsables de los procesos, y se establecen procedimientos para su gestión. Pero existe la necesidad de definir mejor los objetivos de los procesos, y una mayor concreción y elaboración de las estrategias.

\section{Resultados en los usuarios}

Destaca positivamente la disponibilidad de todo el personal del centro, equipo directivo, personal de administración y servicios, profesores, tutores para atender a padres y a alumnos, tanto para su información como para recibir sus quejas. Se ve la necesidad de captar nuevos alumnos mediante una más cuidada proyección de los resultados que consigue el Centro. Y mejorar la movilidad de los estudiantes mediante convenios de intercambio con países europeos, y participación en proyectos y concursos para mejorar la formación de los alumnos. Necesidad de elaborar un plan de formación continua de los alumnos.

\section{Resultados en personas}

Existe una buena relación del personal del Centro con los alumnos y los padres. Necesidad de realizar convenios para una participación en actividades de formación y para ampliar las relaciones con otro centros, otras instituciones y países.

\section{Resultados en Sociedad}

Explorar las necesidades y expectativas de la sociedad en relación con el Centro y atenderlas a través de los criterios agentes. Mejorar las instalaciones del Centro y ofertarlas al entorno próximo.

\section{Resultados clave Centro}

Se valora de modo positivo el cumplimiento de los horarios, de las programaciones didácticas y de la gestión del material y equipamientos. Pero es necesario 
reducir las tasas de repetidores y abandonos, mediante la mejora de las funciones del Departamento de Orientación, con la aplicación de programas de orientación en los distintos ciclos, dirigidos al profesorado, a los alumnos, padres, y personal de administración y servicios. Mejorar y ampliar los tiempos de tutorías en cada uno de los cursos. Realización de planes de acción tutorial y planes de atención personalizada a los alumnos.

Se recomienda la creación de un Departamento de Calidad y el reforzamiento del Departamento de Orientación del Centro.

\subsection{Experiencia en el Centro Educativo 2}

La decisión de evaluar el centro fue planteada en el Equipo Directivo, y llevada al Claustro y al Consejo Escolar para su aprobación. Se decidió contratar los servicios de un consultor experto en el Modelo, de una Auditoria autorizada por el Club Gestión de Calidad. El consultor informó y formó en el Modelo al personal del centro, a su casi totalidad, dado que mostraron interés por formarse y realizar la autoevaluación del centro. El consultor ayudó en la contestación del cuestionario mediante la herramienta Perfil V.4.0, con el fin de que se entendiera de manera precisa la finalidad de cada una de las preguntas. También asistió a las reuniones para consensuar los resultados, y posteriormente ayudó a la elaboración del informe de la autoevaluación. Mostramos los resultados obtenidos.

\begin{tabular}{|ll|l|}
\hline \multicolumn{2}{|c|}{ Perfil del Centro 2} \\
\hline Criterios & Valoración Media \% \\
\hline 1. $\quad$ Liderazgo & 43 \\
\hline $2 . \quad$ Política y Estrategia & 38 \\
\hline $3 . \quad$ Personal del centro & 40 \\
\hline $4 . \quad$ Colaboradores y Recursos & 36 \\
\hline $5 . \quad$ Procesos & 35 \\
\hline 6. & Resultados en Usuario & 28 \\
\hline $7 . \quad$ Resultados en Personas & 27 \\
\hline $8 . \quad$ Resultados en Sociedad & 29 \\
\hline $9 . \quad$ Resultados Clave Centro & 26 \\
\hline Total & 33,55 \\
\hline
\end{tabular}

Los valores obtenidos en los criterios del bloque de los resultados no son muy elevados, especialmente los referidos a los usuarios, alumnos y familias y a las personas del centro. Esto indica que debemos mejorar las acciones de los criterios del bloque de agentes o facilitadores, comenzando por el Liderazgo, que si bien obtiene la mayor puntuación de mostrar más decididamente su compromiso con objetivos de calidad, incluidos el rendimiento de los alumnos y la formación del personal docente. Definición de la Política y la estrategia y la elaboración del Proyecto Educativo del Centro, en coherencia con ellas. Una gestión basada en procesos, alineados 
con la política y la estrategia consensuados por el Claustro y el Consejo Escolar. Desarrollo del Departamento de Recursos Humanos, para llevar a cabo análisis de la formación necesaria del personal, para abordar con éxito los procesos. Análisis de los recursos necesarios y de los colaboradores externos. Concreción de los procesos, y nombramiento de los responsables de cada proceso, y de sus colaboradores, así como de las estrategias, temporalización para su aplicación, criterios para su seguimiento, e indicadores de medida y evaluación.

Es conveniente, para garantizar el desarrollo de la política de calidad del centro, la creación de un Departamento de Calidad, integrado por un equipo de personas interesadas en comprometerse con la gestión de calidad del centro, la elaboración de los planes de mejora, y la autoevaluación continua de los mismos.

\subsection{Experiencia en el Centro Educativo 3}

El objetivo de la evaluación del centro es analizar su funcionamiento en relación con los principales elementos del Proyecto Educativo, y siguiendo las indicaciones del Modelo de Excelencia de la EFQM. Mostramos el perfil del centro:

\begin{tabular}{|ll|l|}
\hline \multicolumn{2}{|c|}{ Perfil del Centro 3 } \\
\hline Criterios & Valoración Media \% \\
\hline $1 . \quad$ Liderazgo & 57 \\
\hline 2. $\quad$ Política y Estrategia & 56 \\
\hline 3. $\quad$ Personal del centro & 30 \\
\hline $4 . \quad$ Colaboradores y Recursos & 34 \\
\hline $5 . \quad$ Procesos & 36 \\
\hline $6 . \quad$ Resultados en Usuario & 52 \\
\hline $7 . \quad$ Resultados en Personas & 36 \\
\hline $8 . \quad$ Resultados en Sociedad & 56 \\
\hline 9. $\quad$ Resultados Clave Centro & 50 \\
\hline Total & 45,22 \\
\hline
\end{tabular}

En relación con los valores máximos del modelo, los porcentajes obtenidos y que se muestran en el perfil del centro, han resultado altos. No obstante, las respuestas en los subcriterios del cuestionario permiten la detección de puntos débiles y fuertes con detalle.

Así, encontramos que son cinco los criterios que obtienen un valor superior al 50 \%. Son: Liderazgo, Política y estrategia, Resultados en la sociedad, Resultados clave del centro, y Resultados en los usuarios. Muestra gran coherencia entre las valoraciones dadas a los criterios agentes y a los criterios resultados. Así, con un Liderazgo comprometido con objetivos de calidad, una Política y estrategia bien definida y consensuada, unos Procesos relativamente bien gestionados, conllevan a unos Resultados en la sociedad positivos, así como los Resultados clave del Centro y los 
Resultados en los usuarios. También se da una gran correlación entre el Personal y los Resultados en el personal, y en la Gestión de recursos, con puntuaciones bajas. Esto puede deberse a que el $20 \%$ de la plantilla del centro no es fijo.

Entre las mejoras a llevar a cabo figuran una mejor información a todo el personal sobre los proyectos institucionales, y especialmente se quiere mejorar el plan de acción tutorial con los alumnos, mediante programas de atención personalizada. Asimismo se quiere trabajar, con la colaboración de la administración educativa, en el establecimiento de relaciones con centros educativos de la comunidad con el fin de compartir proyectos y aprender de las mejores prácticas realizadas en los mismos. La presentación de proyectos para el desarrollo de las nuevas tecnologías en el centro, para obtener ayudas económicas y de formación.

\subsection{Experiencia en el Centro Educativo 4}

En este centro se viene trabajando en el desarrollo de una cultura de calidad desde hace dos años, con la colaboración de un experto externo que ha realizado un curso extensivo e intensivo para formar a todo el personal del centro, y especialmente al Equipo de Calidad. Tras la formación en el modelo, 9 miembros del centro han evaluado la gestión del mismo mediante el formulario, obteniendo el siguiente perfil del centro:

\begin{tabular}{|ll|l|}
\hline \multicolumn{3}{|c|}{ Perfil del Centro 4 } \\
\hline Criterios & Valoración Media \% \\
\hline 1. & Liderazgo & 42 \\
\hline 2. & Planificación y estrategia & 31 \\
\hline 3. $\quad$ Personal del centro & 40 \\
\hline $4 . \quad$ Colaboradores y Recursos & 32 \\
\hline $5 . \quad$ Procesos & 44 \\
\hline 6. & Resultados en Usuario & 28 \\
\hline $7 . \quad$ Resultados en Personas & 14 \\
\hline 8. & Resultados en Sociedad & 22 \\
\hline 9. $\quad$ Resultados Clave Centro & 36 \\
\hline Total & 33 \\
\hline
\end{tabular}

Los resultados aportan valoraciones positivas acerca de la definición de la misión y valores del centro están clara y explícitamente, contando con unos valores propios que los diferencian de otros centros. Se elaboran los presupuestos anuales, y se cumplen. Se analizan y anticipan las necesidades de los alumnos para acceder al mercado laboral o continuar sus estudios y se elaboran planes complementarios para satisfacer las nuevas necesidades detectadas en los usuarios. El Centro involucra a las familias en las actividades educativas y formativas de los alumnos. Y establece colaboraciones en proyectos con otras organizaciones. Consigue los objetivos 
académicos. Se asegura que se implanta la mejora continua a todos los niveles y en todas las actividades del centro.

Las áreas de mejora detectadas en el análisis del proceso y de los resultados, llevan al Equipo de Calidad a priorizarlas de acuerdo con su gravedad en relación con el impacto en los usuarios, en la organización, y su viabilidad. Mencionamos algunas de ellas:

- Mejorar las técnicas pedagógicas de los profesores.

- Concretar un sistema de evaluación del personal, a través de indicadores, para que los líderes reconozcan los esfuerzos y logros de las personas.

- Mejorar, mediante incentivos, la implicación del personal en la mejora del centro.

- Identificar los procesos claves que permitan alcanzar los objetivos del centro.

- Analizar las mejores prácticas de otros centros como estrategia de aprendizaje.

\section{CONCLUSIONES SOBRE LA EVALUACIÓN DE LOS CENTROS EDUCATIVOS}

Las ventajas del Modelo de Excelencia Europeo como ayuda al conocimiento del centro, aportando un informe diagnóstico con sugerencias para la realización de Planes de Mejora son evidentes. Como herramienta de autoevaluación no supone costes adicionales, si bien para su mejor aplicación se recomienda la contratación de un experto en el Modelo que ayude a formar a los miembros del Equipo de Calidad, y a informar y sensibilizar hacia la cultura de calidad a toda la comunidad educativa. La posibilidad de comparar los resultados obtenidos con otros centros, y el beneficiarse de las mejores prácticas, potenciará el éxito de los Planes de Mejora en los centros, y de su calidad.

Los Planes de Mejora se basan en los puntos negativos detectados, y en priorizar las necesidades en función de su mayor gravedad, incidencia en el rendimiento y formación de los alumnos, y plausibilidad de que tengan éxito de acuerdo con los recursos actuales, y tratar de conseguir recursos y mejoras en formación específica para atender a las necesidades señaladas.

Para la puesta en marcha de los Planes de Mejora se considera necesario crear un Equipo de Calidad, o mejor un Departamento de Calidad, interdepartamental, formado por miembros del Equipo Directivo y de los diferentes Departamentos de los niveles educativos y áreas curriculares, que serán los responsables de los procesos y de dinamizar el desarrollo de la cultura de calidad.

Los ciclos de autoevaluación utilizando el Modelo de Excelencia, suelen ser de curso completo, pero pueden realizarse evaluaciones parciales para comprobar los resultados que se van consiguiendo en los planes de mejora específicos, lo que permitirá ver la tendencia de mejora e identificar nuevas áreas de cambio y mejora a 
tiempo. La reducción de los ciclos de mejora contribuirá a un mejor seguimiento de los avances y a la instauración de la mejora continua y de la cultura de calidad en el centro.

La evaluación formativa debe compartir las mejoras en todos los niveles del sistema, desde la evaluación del aprendizaje del estudiante, la evaluación de la mejora de los centros, y la evaluación de la mejora general del Sistema Educativo. La información recogida en cada uno de los niveles del sistema debe ser utilizada para identificar sus fortalezas y debilidades, y compartir las estrategias para la mejora. (CERI/OECD 2005).

Dependiendo de los ámbitos de aplicación de la mejora, se responsabilizará del proyecto a la persona más idónea en ese ámbito, con colaboradores para su aplicación, debiendo concretar los objetivos, los contenidos, las estrategias, los recursos, los tiempos de aplicación, y los criterios para su seguimiento, medición de logro y evaluación. Asimismo, se cuidará la coordinación entre todos los procesos. La escuela, como comunidad de aprendizaje, debe reflexionar en equipo sobre los problemas que le preocupan, para crear las condiciones estructurales para la mejora, centrada, como prioridad, en mejorar el aprendizaje del estudiante, desde una perspectiva de investigación sobre la enseñanza, con el fin de aunar esfuerzos.

Mostramos, en el siguiente diagrama, los objetivos estratégicos para la innovación integral en los centros educativos, desde una perspectiva de síntesis entre diversos enfoques:

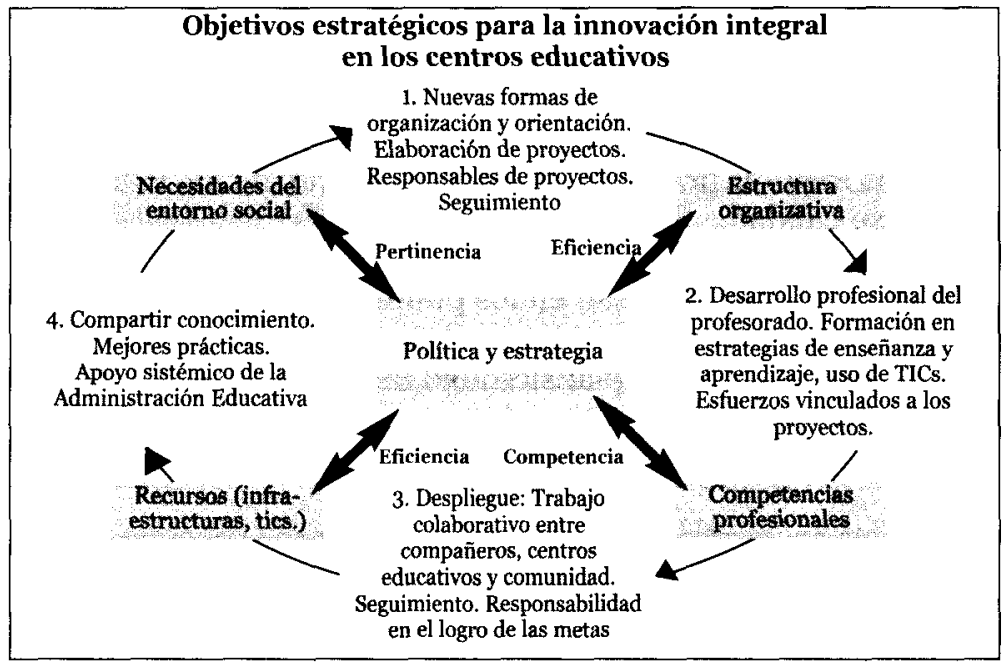

Figura 4. Objetivos para la innovación integral en los centros educativos

Los proyectos, los planes, y las estrategias para su aplicación, su seguimiento y la evaluación de sus resultados, de acuerdo con las necesidades detectadas, deberán estar fundamentados en la investigación, y orientados a la práctica, y centrados en la mejora de aprendizaje y formación del estudiante. Y deberá contar con apoyos 
externos de la Administración educativa, para conseguir colaboraciones específicas. Asimismo, deberán estar coordinados con otros proyectos del centro, y se informará de los mismos al Consejo Escolar, con el fin de que se involucren y se ilusionen en su desarrollo.

En la actualidad hay gran demanda para la realización de proyectos innovativos en relación con la convergencia educativa en Europa, especialmente en relación con la incorporación de las TICs en la enseñanza, al servicio de:

- Una gestión eficaz de la información.

- La mejora de la gestión de los centros.

- La mejora del aprendizaje, especialmente por sus posibilidades para la personalización educativa y el trabajo cooperativo en aula y fuera de ella, y por sus amplias posibilidades para el aprendizaje a lo largo de la vida, en cualquier situación: formativa, laboral, de ocio, y lugar.

Las claves para conducir las mejoras estratégicas en los centros educativos, podemos verlas en el diagrama siguiente, tomado de HOPKINS (2005):

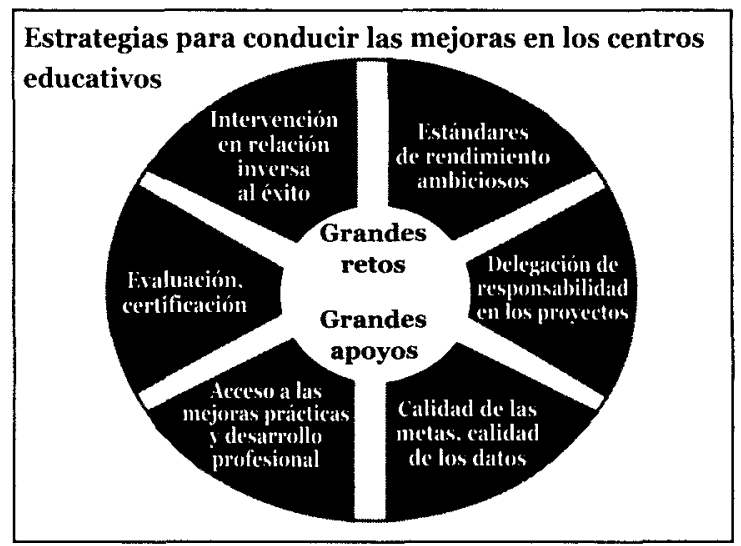

Figura 5. Estrategias para conducir las mejoras en los centros educativos

Los retos deben ser grandes y, en consonancia, los apoyos, debiendo intervenir en aquéllas áreas donde más se necesite, con metas de calidad, orientados a la práctica, buscando resultados ambiciosos de rendimiento en los alumnos, mediante el desarrollo de proyectos fundamentados en las necesidades y en la investigación, informados en las mejores prácticas, con apoyos externos e internos, con responsables para cada proyecto, coordinados, y aplicados de un modo sistémico, atendiendo a la totalidad del centro, con compromiso de servicio a la comunidad, basado en la transparencia que aporta la evaluación mediante constatación de hechos y datos fiables y válidos, para la certificación.

Es de gran importancia el enfoque de evaluación para la mejora, en todos los niveles, basada en hechos y datos. Así, los principios que deben guiar las mejoras en los centros educativos, de acuerdo con (CERI/OECD 2005), son los siguientes: 
- Centrarse en la mejora de la enseñanza y del aprendizaje.

- Alinear los enfoques de evaluación formativa y sumativa.

- Recoger datos sobre el funcionamiento de los centros, en todos los niveles, de clase, centro y sistema educativo, con fines para su utilización para la mejora, con enfoque de evaluación formativa, para compartir las mejoras, sistemáticamente, en todos los niveles.

- Invertir en formación y apoyo para la evaluación formativa.

- Construir fuertes puentes entre la investigación, la política educativa y la práctica educativa.

El interés por la mejora de la calidad de la educación, es compartido en todo el mundo. En el Reino Unido predomina un enfoque distinto al Modelo de Excelencia de la EFQM, con un elevado componente característico de las organizaciones empresariales, orientado a la eficiencia en la gestión. El Reino Unido tiene una mayor tradición en la utilización de enfoques para la mejora en la escuela cercanos a la investigación-acción que desarrollará Stenhouse ${ }^{3}$ y Elliot, y de evaluación interna.

Sin embargo, en la última década, las políticas educativas de los distintos países han sido permeables a la influencia de los principios de la Gestión de la Calidad Total, y de la certificación, o rendimiento de cuentas, como garantía de calidad en la gestión de los fondos públicos:

"El rendimiento de cuentas, en cierto modo, debe ser el fundamento de los Servicios Públicos actuales. Sin rendimiento de cuentas no hay legitimidad; sin legitimidad no hay apoyos; sin apoyos no hay recursos; sin recursos no hay servicios... El rendimiento de cuentas inteligente sirve a dos funciones: ayuda al aprendizaje organizacional, y muestra al público el valor que obtiene por su dinero". (MILIBAND, 2004).

Esta inquietud por la mejora de la Calidad de las Escuelas queda recogida en la declaración de la Secretaría de la Educación Pública de México, en su "Programa de Escuelas de Calidad":

"Una escuela de calidad será aquella que asume de manera colectiva la responsabilidad por los resultados de aprendizaje de todos sus alumnos y se compromete con el mejoramiento continuo del aprovechamiento escolar. Es una comunidad educativa integrada y comprometida que garantiza que los educandos adquieran los conocimientos y desarrollen las habilidades, actitudes y valores necesarios para alcanzar una vida personal y familiar plena, ejercer una ciudadanía competente, activa y comprometida, participar en el trabajo productivo y continuar aprendiendo a lo largo de la vida".

Y asimismo, es importante aunar esfuerzos en beneficio de la mejora, el establecimiento de relaciones mutuamente beneficiosas con otros centros educativos, $y$ organizaciones, lo que facilitará el aprendizaje, y la puesta en práctica, con fundamentos seguros, que ayudaran a su éxito de las mejoras. 
"Si nuestra meta es conseguir la excelencia y la equidad, entonces, la política y la práctica deben centrarse en los sistemas de mejora. Esto significa que el director de un centro educativo tiene que estar tan implicado en el éxito de otros colegios como lo esté en el suyo propio. Las mejoras sostenidas de los colegios no serán posibles al menos que la totalidad del Sistema Educativo se mueva en la misma dirección”. (HOPKINS, 2005).

Por todo ello, será decisivo que la Administración Educativa de nuestro país, España, haga una apuesta clara y comprometida con la "Evaluación de los Centros Educativos" para que la mejora de la calidad de la Educación pueda iniciar un camino seguro, siendo necesaria la creación, en los centros de un Departamento de Calidad, que trabaje unido al Equipo Directivo, y al Departamento de Orientación, con ayudas para la formación de su personal en cuestiones de Calidad.

\section{NOTAS}

1.- www.efqm.org, 2002. El último criterio es: "Resultados clave de rendimiento" de la organización, (2003).

2.- Este apartado está basado en la publicación del MECD (2001).

3.- "It is teachers who, in the end, will change the world of the school by understanding it". Cita de Lawrence Stenhouse elegida por algunos de los profesores que han trabajo con él, para la inscripción en su memoria en el campus de la Universidad de East Anglia. En David Hopkins (2004). Transforming Schools. Key Strategies for Action. Keynote presentation tothe "Transformation through Global Networking". Conference Melbourne, Australia. 27 th July. 


\section{REFERENCIAS BIBLIOGRÁFICAS}

CERI/OECD (2005). Improving Learning through Formative Assessment. 3 February.

Club Gestión de Calidad European Foundation For Quality Management (2003). $\mathrm{El}$ informe de excelencia en España. Madrid.

Confederación Española de Centros de Enseñanza (2000). Guía de Autoevaluación. Adaptada a Centros Docentes y/o de formación. Madrid: CECE, EOQ, EFQM, OGC.

Corbett, C. J., Luca, A. M. (2003). Global perspectives on global Standars. A 15economy survey of ISO 9000 and ISO 14000. ISO Management Systems, JanuaryFebruary (31-40). www.iso.org

Deming, W. E. (1950). Elementary principles of the statistical control of quality. Japanese Union of Scientific and Engineers (JUSE). Tokyo.

EFQM (2003). Introducción a la Excelencia. www.efqm.com

Hopkins, D. (2004). Transforming Schools. Key Strategies for Action. Keynote presentation to the "Transformation through Global Networking". Conference Melbourne, Australia. 27 th July.

Hopkins, D. (2005). Large Scale Reform in England-Towards a High Excellence, High Equity Education System. RM Education in the Future Conference. Manchester, 2nd March.

ISO (2002). Feedback on ISO 9001:2000. Worldwide round-up of early market reactions. ISO/TC 173 Subcommittee 2. ISO Management Systems, JanuaryFebruary, (19-29).

Martínez Mediano, C. (Coord.). (2004). Técnicas e Instrumentos de Recogida $y$ Análisis de Datos. Madrid: UNED.
Miliband, David (2004). Accountability in the school system. Assessment conundrums. Conference of Education, Belfast.

Ministerio de Educación, Cultura y Deporte. (2001). Modelo Europeo de Excelencia. Adaptación a los centros educativos del modelo de la Fundación Europea para la Gestión de Calidad. Madrid: MECD.

Secretaría de Eduación Pública de México. (2002). Programa de Escuelas de Calidad. México.

Shewhart, W. A. (1939). Statistical method from the viewpoint of quality control. Graduate School. Washington, D.C.: Department of Agriculture.

Varios (2005). Calidad en educación, calidad de la educación. Documentos para una concepción integral e integrada. Madrid: AEC.

\section{DIRECCIONES DE INTERNET}

www.aec.es

www.aenor.es

www.baldrigue.org

www.cece.org

www.clubcalidad.org

www.deming.org

www.efqm.org

www.fundibeq.org

www.iso.org

www.juraninsitute.org 


\section{PALABRAS CLAVE}

Calidad en educación. Modelo Europeo de Excelencia aplicado a los centros educativos.

\section{KEY WORDS}

Quality in education. European Excellence Model applied to schools.

\section{PERFIL ACADÉMICO DE LAS AUTORAS}

Catalina Martínez Mediano es Profesora Titular. Imparte las asignaturas de Evaluación de Programas y Técnicas e Instrumentos de Recogida y Análisis de Datos, en las carreras de Pedagogía y Educación Social, y diversos cursos de doctorado. Viene trabajando en Evaluación de Programas y Modelos de Calidad aplicados a los Centros Educativos. Participa en investigaciones sobre plataformas educativas en internet, en la evaluación de su eficacia pedagógica.

Nuria Riopérez Losada es Profesora Ayudante Doctora. Es profesora del Practicum de Educación Social y Pedagogía, e imparte la asignatura de Técnicas e Instrumentos de Recogida y Análisis de Datos, y diversos cursos de doctorado. Viene trabajando en Educación Multicultural y Educación Especial, y participa en diversas investigaciones sobre estos campos.

Dirección de las autoras: Dpto. MIDE I Facultad de Educación, UNED

Paseo Senda del Rey, 7 28040 Madrid

Fecha recepción del artículo: 30. marzo. 2005

Fecha aceptación del artículo: 18. junio. 2005 